\title{
Fertility preservation options in gender reassignment
}

39\% of transgender men and
$21.9 \%$ of transgender women
express the desire to become
parents. This is complicated by
gender confirmation therapies
usually occurring at a young
age, before consolidation
of parental desire. These
therapies can involve hormone
treatment and gender
confirming surgery which
can remove the possibility
of reproduction. Fertility
preservation knowledge and
information is limited for
transgender people. Kelly
Tilleman, the director of the
In-Vitro Fertilisation (IVF)
laboratory at Ghent University
Hospital, Belgium and her
colleagues explore the options
available to transgender and
gender diverse people to
ensure accurate information
is given prior to gender
confirming treatments.

E tranility preservation and rapidly growing areas of research and clinical practice. The Transgender Health (WPATH) Standard of Care recommends in their seventh version that people seeking gender confirmation treatments are made aware of their fertility preservation options before undertaking any treatment. At the current time, thoug there is limited knowledge on the options available and their feasibility for transgender people, and those supporting them.

Currently the established fertility services options for transgender men are oocyte cryopreservation and in case of a partnership embryo cryopreservation. For transgender women, sperm cryopreservation is the established possibility of cyopresenving gono the possibility of cryopreserving gonadic

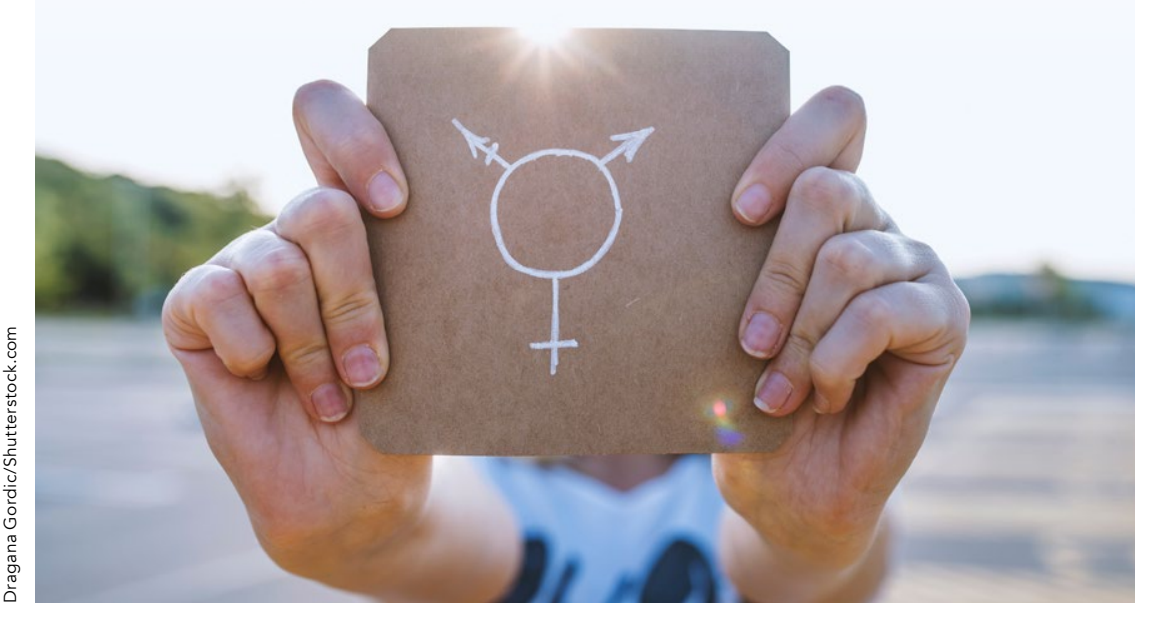

and are hence not the established fertility preservation method of choice.

\section{PARENTAL DESIRES}

Half of transgender men have been more recent and focused studies

identified that $39 \%$ of assigned female at birth and $21.9 \%$ assigned male at birth respondents had parental desires. Whilst these contemporary figures are lower, they still indicate that a large proportion of transgender people desire to have children. Parental desires may be achieved through social or biological methods such as adoption of children prior to or post gender confirmation treatments. surrogacy (in some regions) or accessing fertility services for assisted eproduction techniques.

All options have a wide range of issues to consider. Barriers identified by fulfilment of parental desires include concerns related to the adoption procedure and prejudices that may be experienced within it They perceive that these prejudices will also cause any child they may have will be discriminated against and that they may as a transgender parent experience
discrimination. Whilst transgender people may be fearful of discriminatio they are also concerned that to fulfil parental desire using their own genetic material may be extremely costly financially. Social methods of achieving parental desire such as adoption and surrogacy are not considered here but are options that need further
discussion. The biological fertility

reatment options are explored by her colleagues and described here. For persons assigned male at birth (AMAB) the fertility preservation option of freezing sperm (sperm cryopreservation) has been available through fertility services since the 1950s, is effective and considered easy to perform. Despite this, it is important to note the potential psychological burden as it may not be easy for AMAB to collect a semen sample by masturbation. Sperm can also be collected and frozen prior to any gender confirmation treatmen through surgical extraction but this is a surgical
associated risks.

The remaining option is theoretical and experimental and involves the surgical collection of testicular tissue which is frozen with a view to unfreeze and mature later either in vitro or through transplantation followed by assisted reproduction techniques. The use of testicular tissue to obtain

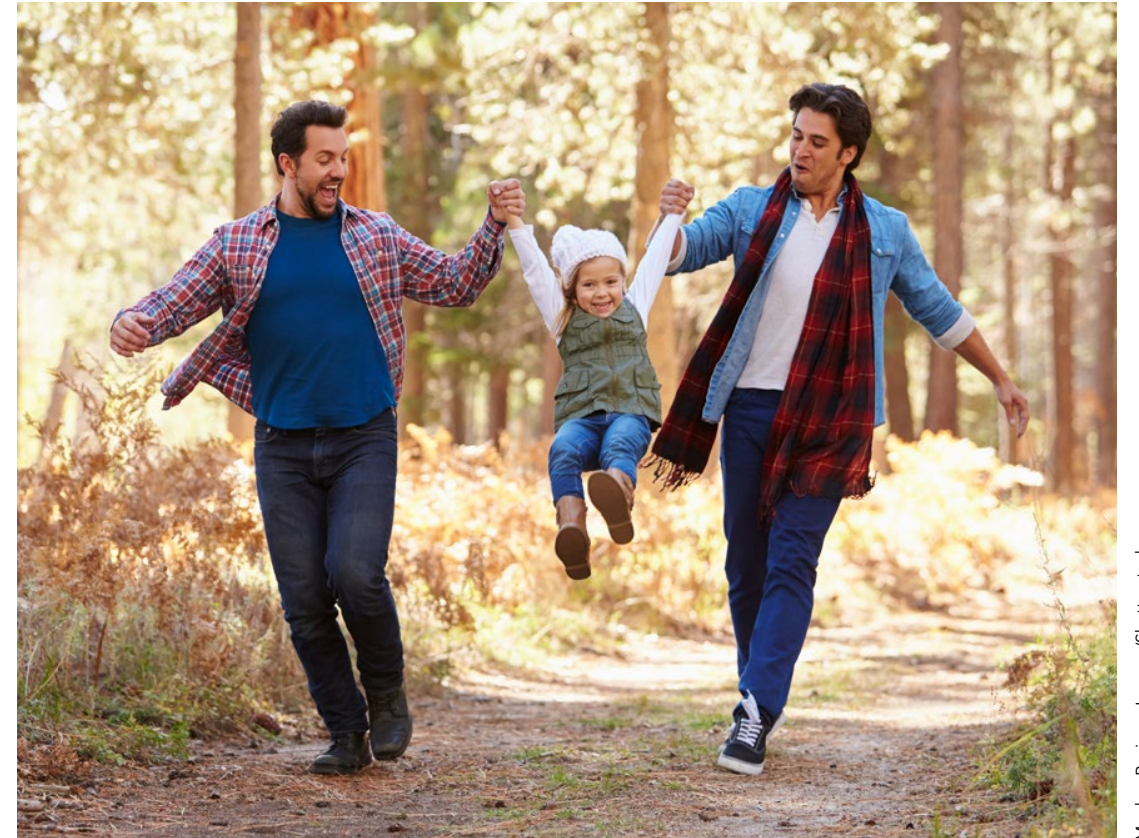

Recent studies identified that $39 \%$ of assigned female at birth and $21.9 \%$ assigned male at birth respondents had parental desires. mature spermatozoa has never been

BIRTH ASSIGNED FEMALES As is the case for AMAB, the fertility preservation options for persons assigned female at birth (AFAB) are limited and each approach needs careful consideration prior to any interventions being ocyte cryopreservation, embro cryopreservation and the experimenta ovarian tissue cryopreservation. Each are fraught with challenges.

Oocyte cryopreservation requires the AFAB ovaries to be stimulated and mature oocytes to be collected. This during the period of ovarian stimulation, wishis to cor tim their male gerwor.

Embryo cryopreservation involves the harvesting of mature oocytes which ca requires vaginal ultrasound monitoring

be fertilized in vitro. An early decision sperm sample used in the fentization is acquired.

The third option is experimenta equiring the collection of ovarian tiss which contain follicles with immature oocytes. The tissue is then frozen ready in the future. This techniqued $n$ vitio theory not require a postponay, the gender confirming thera geutical

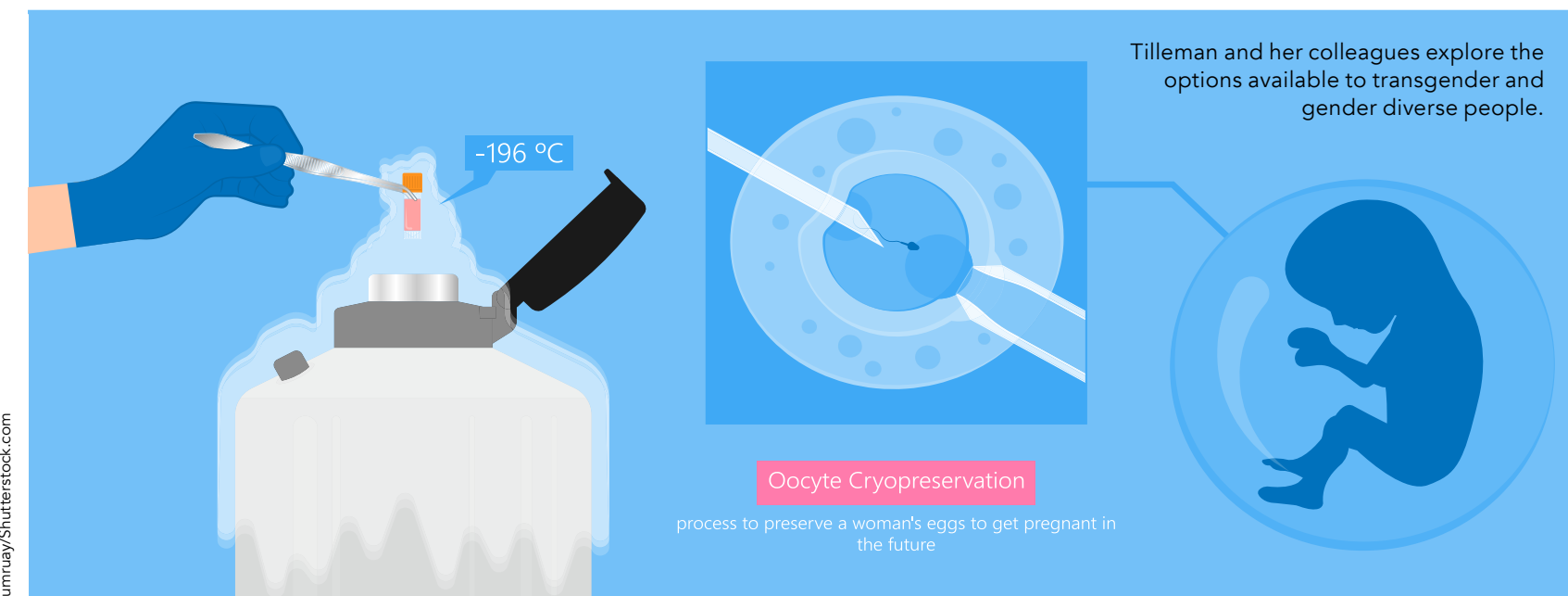




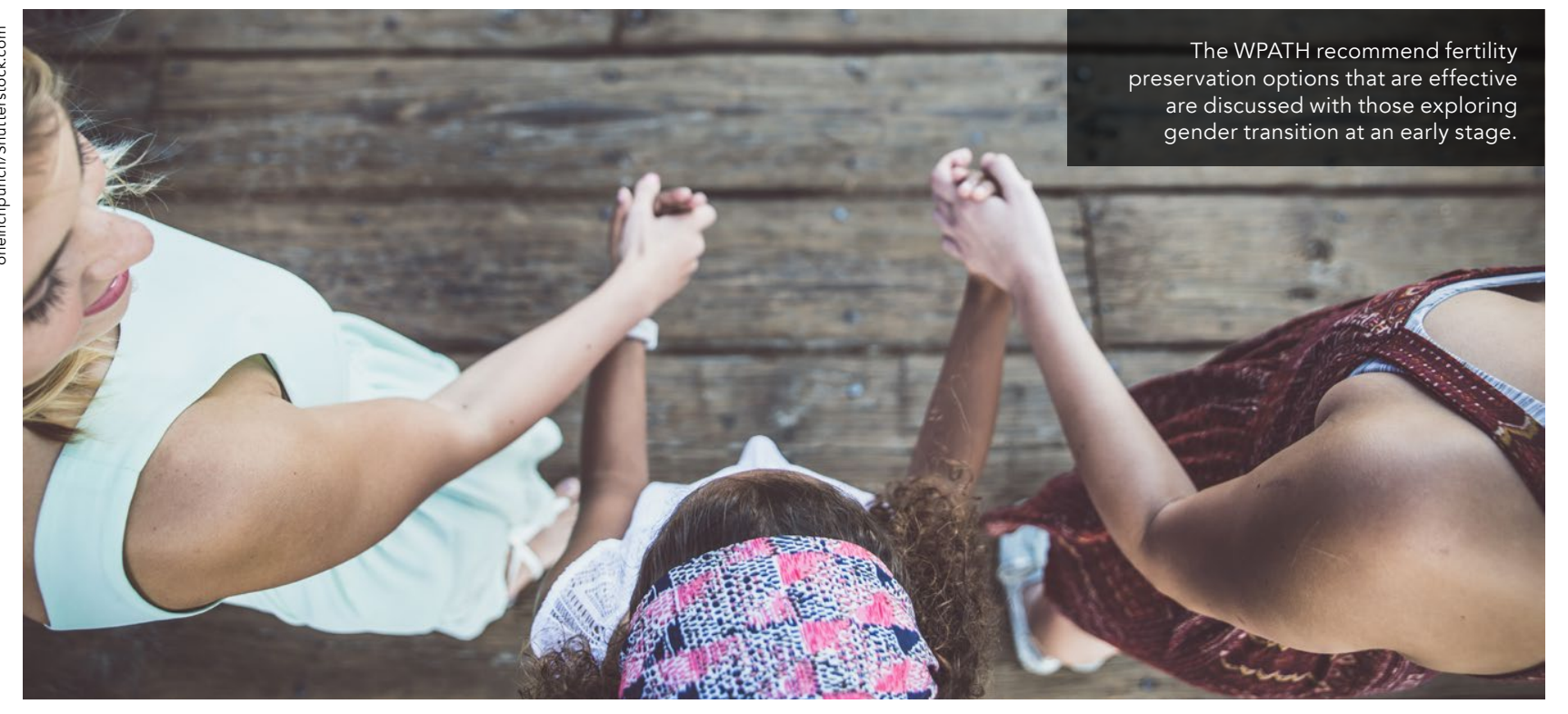

be undertaken after commencement

(TOLAN IMMATURE OOCYTES)

COLLECTIONOFOVARIANTISS

This ex-vivo method is already used

women commencing cancer treatment

which may make them infertile. The

use of ovarian tissue (to gain immatu

oocytes) for fertility preservation

in women with cancer has been a

remains experimental, especially during testosterone therapy. Tilleman and colleagues have undertaken detailed
studies to establish studies to establish the feasibility of this form of fertilly

preservation.

At present there remain limited options for fertility preservation for transgender and gender

ovarian tissue for collection of immature oocytes could, for AFAB, be undertake during oophorectomy as part of gender confirmation surgery. This would reduce medical intervention during their gender confirming journey and remove the obstacles associated with mature oocyte collection to facilitate fertility preservation. This also removes the need to make parental decisions before the person has established their parental desires.

OVARIAN TISSUE AFTER

TESTOSTERONE THERAPY different in AFAB wos (OTO) are testosterone therapy to those diverse people.

undergoing oncology treatment. The ovarian cortex is stifer in AFAB, desp oocytes emerge, are not destroyed.

cevity and outer cells, had developed. Abnormal cleavage (developmenta) collected observed in $91.7 \%$. Genetic analysis as cumulus-oocyte-complexes (COCs) from within the follicles of the ovarian tissue. After these COCs have been collected from transgender men usually at the time of oophorectomy and hysterectomy they were in vitro red. These in vitro matured COCs were examined for spindle structure and chromosomal alignmen observed in $91.7 \%$. Genetic analysis chromosomal patterns in $42 \%$ of the embryos analysed.

\section{CONCLUSION}

Despite fertility preservation and transgender healthcare research and clinical practice rapidly growing at present there remain a few

time limited options for fertility

perseveration for transgender people. The WPATH recommen fertility options that are after freezing; they have been shown to have normal meiotic spindles. Unfortunately, the ovarian tissue oocytes in vitro matured (OTO-IVM) during testosterone treatment, show very low fertility feasibility.

\section{OTO-IVM}

In their recent publication, Lierman et al. found the OTO after warming survival rate to be $72.6 \%$ but the fertilisation rate of these oocytes w reached day 3 with more than or equal to 6 cells. By day 5 only one three parts, inner cells, fluid filled

effective are discussed with those exploring gender transition at an early
stage. Given current knowledge if this is not undertaken at the beginning of the gender confirming journey, before or after cessation of the cross hormone treatment, transgender people may lose the opportunity for fertility by the time they identify parental desires.

For AMAB sperm cryopreservatio is the only established fertility given the current research knowledge available there are realistically only or embryo cryopreservation. only $34.5 \%$. Half of these embryos preservation technique. For AFAB

\section{(f) Behind the Research}

\section{Dr Kelly Tilleman}

E: Kelly.Tilleman@UZgent.be W: $\mathbf{w w w}$. epath.eu W: $\mathbf{w w w}$ wpath.org $\mathbf{W}$ : www.transgenderinfo.be

\section{References}

Lierman S, et al. 2021. Low feasibility of in vitro matured during ovarian tissue preparation at the moment of gender confirmation surgery and during testosterone treatment for fertility preservation in transgender men
Fertil Steril. S0015-0282(21)00214-4. doi: 10.1016/i. ertnstert 202103009 . Online ahead of pint-

Defreyne J. et al. 2020. Parental desire and fertility preservation in assigned male at birth transgender people living in Belgium, Int. J Trans Healh to

Defreyne J, Van Schuylenbergh J, Motmans J, Tilleman $K$, 'SSjoen G. 2020. Parental desire and fertility preservation in assigned female at birth transgender people living in

Lierman S, Tilleman K, et al. 2017. Fertility preservation for trans men: frozen-thawed in vitro matured oocytes exhibit normal meiotic spindles. J Assist Reprod Gent. 2017;34(11):1449-56

De Roo C, Lierman S, Tilleman K, et al. 2017. Ovarian people: insights into ovarian histology and physionder after prolonged androgen treatment. Reprod Biomed Online. 2017;34(6):557-66

De Roo C, Tilleman K, T'Sjoen G, De Sutter P. 2015. Fertility options in transgender people, International Review of Psychiatry,
doi:10.3109/09540261.2015.1084275

Segers I, et al. 2015. In vitro maturation (IVM) of oocytes recovered from ovariectomy specimens in the laboratory
a promising "ex vivo" method of oocyte cryopreservatio resulting in the first report of an ongoing pregnancy in
Coleman E, et al. 2012 Standards of care for the health of transsexual, transgender, and gender nonconforming people, version 7. Int J Transgenderism. 2012;13(4):165-232 Wierckx K, et al. 2012. Reproductive wish in transsexual men. Hum Reprod. 2012;27:483-7.

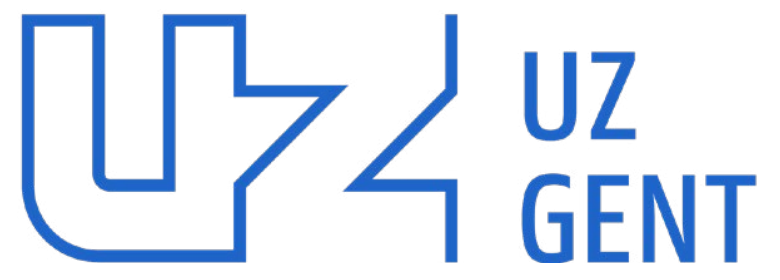

\section{Detai}

Address

Chent University Hospita

B-9000 Ghent

Belgium

The IVF laboratory Ghent University Hospital performs all IVF d reproduction, and run a successful fertility preservation program.

Kelly Tilleman's research experience started in the field of proteomics where she obtaned a PhD in biomarker research She combined her scientific enthusiasm with the responsibility of quality manager for an accredited DNA fingerprinting laboratory. As a post-doctoral fellow Dr Tilleman collaborated with Prof Petra De Sutter and began working at the fertility center with Prof De Sutter in 2009 where her research in the persons began.

Currently, Kelly Tilleman is director of the IVF laboratory at Ghent University Hospital. Internationally, she is coordinator

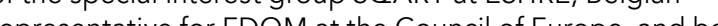
member of international organization ICCBBA (ISBT-128).

Funding

Collaborators

- Promotors of my research: Petra De Sutter (department of reproductive medicine - Ghent University Hospital) and Guy Sjoen (department of endocrinology - center for sexology and gender, $G$ hent Belgium)

medicine- Ghent University Hospital) of reproductive

\section{Personal Response} II We are in the process of drafting a research protocol for
AFAB to harvest ovaries before testosterone treatment. At this time, the ovaries have not been exposed to testosteron yet. We can use these donations for optimisation of the
OTO-IVM technique. If this would be found worthwhile, it accompanied with endovaginal ultrasound monitoring, and
result in mature and fertilisable oocytes. 